\title{
Recurrent Difficulties: Solving Quantitative Problems
}

\author{
H. Kramers-Pals \\ Department of Chemical Technology, Twente University of Technology, P.O. Box 217, 7500 AE Enschede, The Netherlands
}

\section{J. Lambrechts and P. J. Wolff}

Laboratory School, Hengelo, The Netherlands

Quantitative problems are a major impediment for students in chemistry courses, both at the secondary and the tertiary levels. In tests and exams, $p$ or $p^{\prime}$ values ${ }^{1}$ of 0.50 or lower occur frequently. These results are disappointing because of the amount of time and energy students spend in class and at home on learning how to solve quantitative problems. We, therefore, considered it worthwhile to try to improve the way students solve these problems. We would like to teach students to solve these problems more systematically, with fewer errors and faster-in short, more successfully.

Our work has been inspired by the work of Mettes and Pilot on problem solving at Twente University of Technology (TUT) (1-3). They have developed a system of heuristics for solving problems in science, derived from a Program of Actions and Methods (PAM). They have also developed a plan of instruction to teach this PAM. Both PAM and the plan of instruction have already proved useful for several courses at university level: Thermodynamics (1), Electricity and Magnetism (4), and Mechanics.

In our opinion, both PAM and the plan of instruction contain many useful features for the teaching of chemistry and physics in lower-level courses. To check the usefulness of PAM, we chose the first year course in general chemistry taken by students training to be laboratory technicians. ${ }^{2}$

In this paper, recurrent difficulties encountered by students in this course will be analyzed with the help of a simplified version of PAM. This analysis will help us to decide which parts of PAM will need more, or different, emphasis in teaching and exercising.

For this analysis, most information was collected by having students solve problems while thinking aloud. We will pay some attention to this useful and simple method of collecting data on students' problem-solving behavior.

We will also discuss some noticeable aspects of the problem-solving behavior of teachers. Many students' difficulties can be traced to this behavior. In comparison to students, teachers are experienced problem solvers. Moreover, almost all problems that students have to solve in class are routine for the teachers. Hence, their problem-solving behaviors are different. Teachers may pay too little explicit attention to several phases of the problem-solving process that are essential to beginners.

\section{Collecting Data}

Before developing instruction to improve student prob-

\footnotetext{
${ }^{1}$ The value of the facility index $p$ (and $p_{1}$ ) is found by dividing the average score by the maximum possible score on the question. In multiple-choice tests, $p$ is used; $p_{1}$ is used in open examinations. Any book on educational measurement (i.e., Thorndike, R. L., "Educational Measurement," American Council on Education, Washington, 1971) can be used for further explanation of $p$.

2 These students were training at the MBO level. MBO stands for Middelbaar BeroepsOnderwijs (secondary vocational education). The entrance requirement is MAVO (intermediate general secondary education). The age at entrance is about 16 . The MBO curriculum takes three years, including six months' training in a joblike situation. Graduates work in chemical and medical laboratories on routine operations, mainly under supervision.
}

lem-solving behavior, one has to know what this behavior is. Any shortcomings in such behavior must be detected so that they may be remedied.

When students work through problems in class or in tests (and exams), mistakes in the results are evident to the teacher. However, the process of thinking that creates the mistakes is much less evident. Some phases in problem solving, like analysis of data and evaluation of results, are usually only mental processes, if they take place at all. Thus, written student work is only of limited value to the analysis of problemsolving behavior.

With this in mind, we collected most of our information on student problem-solving behavior by having students solve problems while thinking aloud as much as possible.

We used the analysis of tests and exams only as a check on the analysis of "thinking aloud" problem solving. This check, of course, was limited to those phases of problem solving that were written out by the students.

This project and others $(2,4,7)$ have shown that "thinking aloud" problem solving is a very useful method for discovering what may be happening in the student's mind. The method is simple: A few students (or teachers) are asked to participate in the experiment, which will take each of them an hour at the most. One or more relevant problems are chosen. The experimenter and the experimental subject work in a room where they cannot be disturbed. Possibly a third person is present to operate the tape recorder and to record tape numbers corresponding with the subject's notes. The experimenter concerns himself only with stimulating the subject to think aloud. $\mathrm{He}$ gives clues only when the subject reaches an impasse. He should avoid teaching during the experiments but should feel free to give feedback afterwards.

The problem-solving process can be observed directly when students try to solve the problem while thinking aloud. The tape can be studied more thoroughly later. When it is considered worthwhile, the tape recording may be transcribed; the result is called a protocol. Of course, the "thinking aloud" method has some disadvantages:

1) Verbalizing thought may hinder the thinking process, or vice versa.

2) Not all steps in the problem-solving process are verbalized, for several reasons:

- Verbalizing is not easy.

- Not all steps are taken consciously.

- One can think faster than one can speak.

This difficulty can partly be solved by asking the problem solver for an explanation in case of incompleteness.

3) The problem solver finds himself in an unusual situation because of the tape recorder and the presence of the experimenter; even if this is the teacher, the circumstances are different.

In Figure 1, an English translation of one of our protocols is given. Of course some of the colloquial flavor has been lost in the translation.

We asked students of different abilities to solve two problems each. The first problem (given in Fig. 1) was a type with which students had already had some experience. The other problem was on subject matter which was going to be dis- 
cussed in class about a week after the experiment but which the students were already able to understand. The subject matter was limited to titration, mainly because this was the subject matter treated in the class at the time when we were running our experiment. After analysis of the results, the teachers considered the difficulties to be representative of most difficulties first-year students have with quantitative problems in general chemistry. In general terms, the observed difficulties were remarkably similar to those found at different levels and/or with different subject matter $(2,4,7,9)$. A reason for this may be the difference in problem-solving behavior between students and teachers (experts), which we will discuss later.

\section{Method of Data Analysis}

For the analysis of the protocol data (and later of the test examination data), we used a simplified version of PAM. For a more detailed description of PAM, we refer readers to Mettes et al. (2). The principal phases of PAM can be summarized in the model given in Figure 2. A more detailed scheme can be found in the paper by Mettes et al. (1b). We analyzed the protocol data by locating the difficulties students encounter when solving problems in the different phases of this model. The following section will give the results of this analysis. The difficulties encountered will be described in general terms; examples will be given from the protocols and the examinations.

\section{Difficulties Encountered by Students}

\section{Difficulties in Analyzing the Problem}

In this phase, the problem solver should get an overall picture of what the problem is. He should first understand the problem well before he starts to solve it. For a beginning problem solver, this means using paper and pencil (or pen), writing down the data, the unknown, and an estimation of the answer. If possible, these should be ordered, e.g., in a scheme, and written down in correct symbols and units. Difficulties of students in this phase are

- They fail to find all the data by not reading thoroughly enough.

- They start too soon.

- They do not know exactly what the unknown is.

- They do not make an estimation of the answer.
The problem

In order to determine the normality of a $\mathrm{NaOH}$ solution (approximately $0,1 \mathrm{~N}) 1.570 \mathrm{~g}$ of oxalic acid is weighed and dissolved in distilled water. The solution is transferred to a $250 \mathrm{ml}$ measuring flask, filled up and mixed thoroughly. $25 \mathrm{ml}$ of this solution is pipetted into a conical flask. 3 drops of phenolphthaleine indicator solution are added. For titration, 20,00 $\mathrm{ml}$ of the $\mathrm{NaOH}$ solution are needed. Calculate the normality of the $\mathrm{NaOH}$ solution.

Oxalic acid is $(\mathrm{COOH})_{2} .2 \mathrm{H}_{2} \mathrm{O} ; \mathrm{C}=12.0 \mathrm{H}=1.00 \mathrm{O}=16.0 \mathrm{Na}=23.0$

This problem was taken from the collection of problems, used in this school. The reader is advised to solve the problem first before reading the protocol. In the protocol, $\mathrm{E}$ is the experimenter, and $\mathrm{S}$ is the experimental subject.

(S reads problem. Starts writing)

E What are you writing down first?

S I am writing down the data in order to know them. $1.570 \mathrm{~g}$ of $(\mathrm{COOH})_{2} \cdot 2 \mathrm{H}_{2} \mathrm{O}$. You have $250 \mathrm{ml}$ of it, and that corresponds to $20 \mathrm{ml} \mathrm{NaOH}$.

(writes down) $-25 \mathrm{ml}=20 \mathrm{ml} \mathrm{NaOH}$

E What are you doing now?

$\mathrm{S}$ To get the normality of the $\mathrm{NaOH}$ solution I divide grams of oxalic acid multipled by the number of equivalents, by the molar mass of oxalic acid. And then this $25 \mathrm{ml}$, that is $1 / 40$ part of a litre, so one should take the molar mass times 40 . So then, it is:

$$
\frac{1.5750 \times 2}{126 \times 2}
$$

13 E 126 you recall from memory?

$14 \mathrm{~S}$ Yes, because we have been doing this many times.

$15 \quad \mathrm{v}_{1} \mathrm{~N}_{1}=\mathrm{v}_{2} \mathrm{~N}_{2}$

$\begin{array}{lll}16 & 25 & 20\end{array}$

17 Then here $\mathrm{N}_{1}$ is the normality of that oxalic acid solution. That

18 is what we have to calculate first. I make milliequivalents of it.

19 It is 0.625 . Then $\mathrm{N}_{2}: \frac{25 \times 0.625}{20}$ (uses calculator)

20 Oh, that is not right.

21 E How do you see it is not right?

$22 \mathrm{~S}$ The problem says approximately $0.1 \mathrm{~N} \mathrm{NaOH}$.

23 E What was your answer?

$24 \mathrm{~S} \quad 0.8$

$25 \mathrm{E}$ Yes, that is a little too much. It is difficult if you have such an

26 answer. How do you find out in such cases where you made a

27 mistake?

Figure 1. "Thinking aloud" protocol.
$28 \mathrm{~S}$ Usually, I first look it over and when I cannot make it out, I

29 do it all over again. Because usually one just overlooks mistakes.

$30 \mathrm{E}$ And if you do it all over again, do you first read the problem

31 again?

$32 \mathrm{~S}$ Yes.

33 E In this case it might be useful. You missed a datum. That might

34 be your mistake.

$35 \mathrm{~S}$ Yes. I see-here -

$36 \mathrm{E}$ Yes, indeed, you did not use those $250 \mathrm{ml}$.

$37 \mathrm{~S}$ Yes, I did not see that it was filled up to $250 \mathrm{ml}$.

$38 \mathrm{E}$ So you started working too soon.

$39 \mathrm{~S}$ Yes. So this should be 10 ? (changes 40 into 10). If that is right,

40 it should be correct.

41 E Why now that 10 ? Can you explain?

$42 \mathrm{~S}$ Isn't $25 \mathrm{ml}$ the tenth part of $250 \mathrm{ml}$ ? (Uses calculator). Now it is 43 even more.

44 E Then there must be something else, musn't it? There is a problem

45 in one of the following steps. You used all data now, and you also

46 worked out how many equivalents of oxalic acid you have in those

$47 \quad 25 \mathrm{ml}$. altogether.

$48 \mathrm{~S}$ I think this is OK too.

49 E You had $25 \mathrm{ml}$, didn't you? And what does $\mathrm{N}_{1}$ stand for?

$50 \mathrm{~S}$ That is the normality of the oxalic acid.

51 E Yes, and what does the normality means?

$52 \mathrm{~S}$ Oh yes, it is 2,5 milli-equivalents in those $25 \mathrm{ml}$

$53 \mathrm{E}$ Yes, the normality is something else. What is ...

$54 \mathrm{~S}$ That is in a litre. So we should take that times 40 , then the answer

55 is 100 .

56 Then it still is not $\mathrm{OK}$, if we calculate it.

$57 \mathrm{E}$ What do you get now?

$58 \mathrm{~S} 125$.

$59 \mathrm{E}$ That is a lot!

$60 \mathrm{~S}$ (Thinks for a long time)

61 E It was milli-equivalent you said, wasn't it? What do you think

62 the answer is if you multiply that by 40 ?

$63 \mathrm{~S}$ Milli-equivalent. Oh, then it is $\mathrm{OK}$. If one makes equivalents out

64 of those, one should divide by 1000 , then the answer is 0.125 .

$65 \mathrm{E}$ Anyway it is a good thing that you understand you have to check

66 
Reading the problem. Often the students did not read the problem well enough. Accordingly, they failed in finding all data.

Writing Down the Data. Data are written down only fragmentarily. Students mainly write down those data that can be transformed immediately. In our first problem (Fig. 1), this is the transformation of grams of oxalic acid into moles or equivalents, which had been done often in class.

Our second problem started with the sentence "In order to determine the $\mathrm{CaCO}_{3}$ content of limestone, $800 \mathrm{mg}$ of it is weighed and ...."Here, subjects mostly wrote down the 800 $\mathrm{mg}$, but they did not include the limestone. Then they immediately divided $800 \mathrm{mg}$ by the molar mass of $\mathrm{CaCO}_{3}$. This does not make any sense because limestone is impure.

Writing Down the Unknown. Nobody wrote down exactly what the unknown was. In our first problem, this appeared to present no difficulty. But in the more complex second problem, all students ran into difficulties because of this omission. The difficulties would have been fewer if the unknown had been written down in a symbolic form, e.g.,

Unknown: $\mathrm{CaCO}_{3}$ content in limestone; this is $\frac{m_{\mathrm{CaCO}_{3}}}{m_{\text {limestone }}} \times 100 \%$.

Estimation of the Answer: A condition for checking the answer later is that one has an estimate to check against. This does not have to be a numerical estimate; it may be sufficient to state, for example, that the content ought to be between $0 \%$ and $100 \%$, that the concentrated solution has a higher concentration of solute than the dilute solution, or something of this kind. Nobody wrote down an estimation, which could be expected after the omission of the unknown.

\section{Difficulties in Planning the Problem-Solving Process}

As Figure 2 shows, in this phase the problem solver should first establish whether the problem is standard. Actually, a standard problem is no longer a problem because it has essentially been solved: the way to relate unknown to data is known. Only routine operations (mathematical calculations) must be performed to find the numerical answer to the problem.

If the problem is standard for the problem solver, he can go on to the next phase, the execution of routine operations. For the teacher-as an expert-almost all problems at the students' level are standard problems. So he takes one (quick) step in this phase and skips the other steps. Unfortunately, it is those "other steps" that offer many difficulties to students.

If the problem solver does not immediately see the way to solve the problem, he tries to find some possibly useful relations between unknown and data. By applying these relations to the problem situation, he tries to interrelate unknown and data $(6)$.

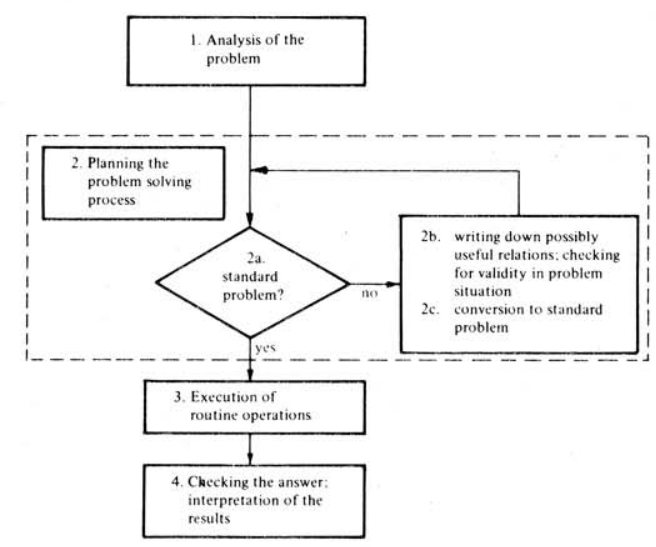

Figure 2. Principal phases of Program of Actions and Methods for systematic problem solving in science (PAM). (1a)
The problem was:

$2.65 \mathrm{~g}$ anhydrous sodium carbonate is exactly neutralised by $12.5 \mathrm{ml}$ hydrochloric acid solution.

Calculate the normality of the hydrochloric acid solution.

We analyzed the answers of 41 students. 17 students had a wrong answer because they used a wrong relation. The 12 wrong relationships are shown, note that several students used the same incorrect relationship. In this analysis, all numbers used by the students are reconverted to the (specified) symbols belonging to quantities of which these numbers give the values. Whenever possible we used the symbols recommended by IUPAC; for equivalents, we introduced the symbol eq, for normality we used $\mathrm{N}$.

Between brackets we give the unit that should have accompanied this combination of numbers. Val is the unit the textbook uses for the number of equivalents.

The right answer is:

$$
N_{\mathrm{HCl}}=\frac{q_{\mathrm{HCl}}}{V_{\mathrm{HCl}}}=\frac{m_{\mathrm{Na}_{2} \mathrm{CO}}}{E_{\mathrm{Na}_{2} \mathrm{CO}}} * \frac{1}{V_{\mathrm{HCl}}}\left(\frac{g}{\mathrm{~g} \cdot \mathrm{Mal}} \mathrm{l}^{*} \cdot \frac{1}{\mathrm{~T}}=\mathrm{val} \cdot \mathrm{l}^{-1}\right)
$$

$$
\begin{aligned}
& \text { (3x) I. } N_{\mathrm{HCl}}=\frac{m_{\mathrm{Na}_{2} \mathrm{CO}_{3}}}{V_{\mathrm{HCl}}}\left(\mathrm{g} \cdot \mathrm{l}^{\prime}\right) \\
& \text { 2. } \mathrm{N}_{\mathrm{HCl}}=\frac{\mathrm{M}_{\mathrm{HCl}} \cdot m_{\mathrm{Na}_{2} \mathrm{CO}_{3}}}{V_{\mathrm{HCl}}}\left(\mathrm{g}^{2} \cdot \mathrm{mol} \cdot \mathrm{l}^{-1}\right) \\
& \text { 3. } N_{\mathrm{HCl}}=\frac{\text { normality }}{V_{\mathrm{HCl}}}\left(\mathrm{val} \cdot \mathrm{l}^{-2}\right)
\end{aligned}
$$

(2x) $4, N_{H C l}=\frac{V_{H C l}}{22.4}($ mol $)$
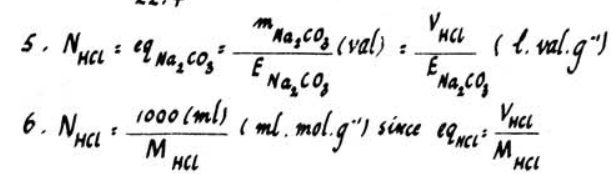

(3x) $7 \cdot N_{H C l}=\frac{V_{H C l}}{M_{H C l}}($ l. mol.g.'

8. $N_{\mathrm{HCl}}=\frac{V_{\mathrm{HCl}}}{1000(\mathrm{mll})}$ (dimmsionless)

9. $\mathrm{N}_{\mathrm{HCl}}=\frac{m_{\mathrm{Na}_{3} \mathrm{CO}_{3}}}{V_{\mathrm{HCl}} \cdot \mathrm{M}_{\mathrm{HCl}}}\left(\operatorname{mol} . \mathrm{l}^{-1}\right)$

10. $N_{\mathrm{HCl}}=\frac{m_{\mathrm{Na}_{2} \mathrm{CO}}}{M_{\mathrm{Na}_{2} \mathrm{CO}_{3}}}, \frac{1}{V_{\mathrm{HCl}}}\left(\mathrm{mol} \cdot \mathrm{l}^{-1}\right)$

11. $N_{\mathrm{HCl}}=\frac{m_{\mathrm{Na}_{2} \mathrm{CO}}}{V_{\mathrm{HCl}}} * E_{\mathrm{Na}_{2} \mathrm{CO}_{3}}\left(g^{2} \cdot v a l^{-1} l^{-1}\right)$

12. 1 mol $\mathrm{Na}_{2} \mathrm{CO}_{3}, \frac{1}{3} \mathrm{val}$

Figure 3. Wrong relationships used by students in solving a problem.

In order to find useful relations, the problem solver should know the subject matter well. He must know what the relations look like. He also must know their validity. Moreover, the subject matter must be accessible to the problem solver: he must know his way in the subject matter so well that he can connect the problem with the subject matter relevant to it. To be more exact, he must have at his disposal the relations that are particularly suitable as a starting point for solving problems. These relations are called key relations. Difficulties encountered by students in this phase are

- They just flit around; they do not work systematically.

- They do not know the subject matter well enough.

- They cannot relate the subject matter to the problem.

Systematic Approach. Several strategies are possible for the approach to a problem. For a "real" problem, use of the unknown as the starting point (backward reasoning) provides a better chance for a successful solution than direct use of the data. The direct use of the data (forward reasoning) offers more chances of transformations that are irrelevant or misleading. Only when the problem is standard is it more efficient to reason forward. 
Although the problems we offered to the experimental subjects were clearly real problems to them, we did not observe any backward reasoning. Forward reasoning is most frequent, although this approach is not chosen on purpose. (In any discussion about an approach or a strategy, it quickly becomes evident that the students are not usually aware of what they are doing.) Backward reasoning is only possible if one realizes what the unknown is. We have seen that this is a difficulty for these students.

Relations and Key Relations. If the student does not know the relation necessary to solve the problem, he becomes completely stuck or will use a wrong relation and thus end up with a wrong answer. In Figure 3, we list all of the wrong relations used by 17 of the 41 students tested. These 17 students either apparently had no key relation available or simply used a wrong one. Quite a few errors arise from the fact that the students ignored the validity of the relations.

In both our problems, almost all subjects ran into difficulties as they applied the relation $V_{1} N_{1}=V_{2} N_{2} \cdot{ }^{3}$ The validity of this relation is limited; it is valid only when both substances involved in the titration are in solution. A better key relation for the titration is $e q_{1}=e q_{2}{ }^{4}$ (at the equivalence point). With the help of the relation $V_{1} N_{1}=e q_{1}$, the relation with more limited validity can be deduced if necessary.

In our protocols, we found that the subjects were aware of applying relations to solve the problems. Those relations they would call "rules." For instance, a student said, "I chose the wrong rule - the same mistake I made some time ago in class."

They also wrote down some rules in symbolic form (e.g., the one mentioned above). This is rather surprising because rules were not offered in this form in class nor in the textbook. The teachers considered a symbolic notation for students at this level too difficult because students supposedly cannot relate the symbols and the physical quantities these stand for.

The difficulties students ran into when using the relation $V_{1} N_{1}=V_{2} N_{1}$ were always the same. They specified $N_{1}$ by filling in the total amounts of matter (in equivalents or milliequivalents). Such a specification error may not only be caused by incomplete comprehension of the relation, but also by an incomplete or incorrect analysis of the problem.

Accessibility of Relations. The number of "rules" students think of when they solve a problem is limited. On solving the second problem, a student said, "Usually, when I have one rule, I do not use another one; otherwise I will mix them up."

When solving the second problem, all subjects needed the clue that the reaction equation might come in handy to connect equivalents and moles in the case of $\mathrm{CaCO}_{3}$. With this clue, they could write down the solution without any further difficulties (except for the equation itself, which surprisingly still offered difficulties).

\section{Difficulties in the Execution of Routine Operations}

Since most students use calculators, calculations offer fewer difficulties than they used to. However, in this phase significant problems remain.

Starting Calculations Too Early. Often students start calculations before they have a good overall picture of the problem situation. The resulting new numbers make the students lose sight of this picture even more.

Omitting Units. Units often are omitted throughout the calculation and suddenly appear behind the final answer. An example of the trouble caused by this is given in Figure 1 .

Omission of the units is not only a source of errors, but is also incorrect. In science problems, physical quantities (e.g., concentration, mass, volume) are taken into consideration.

\footnotetext{
${ }^{3} V_{1}$ : volume of solution $1 ; N_{1}$ normality of solution 1 ; though obsolete, the concept "normality" is still taught in Dutch laboratory schools because of its use in industrial and medical laboratories.

4 eq: symbol used in our project for the number of equivalents.
}

A quantity is a number multiplied by a unit; a number in itself is meaningless in most problems.

By omitting units, students lack the opportunity to check whether the relation applied has been recalled correctly or specified well. (For an illustration, see Figure 3. If the units had been written down alongside the numbers, most students probably would have traced their mistake). It is interesting to read in an American article (8) that students too often solve problems by dimensional analysis only. This is certainly not true for the Dutch students we know.

\section{Difficulties in Checking the Answer and Interpreting the Results}

As we expected, because they omitted an estimate in the analysis phase, students often do not check their answers. We were quite surprised, however, to find that all subjects did check their answer against the approximate answer which was given in the first problem. Even if there is no estimate to check against, students should check the number of significant figures. This check is often omitted, too.

\section{Student Difficulties and Teacher Problem-Solving Behavior}

We believe that many difficulties arise because students were not taught well enough. In fact, many student difficulties can be traced to the way teachers solve problems. In order to explain this, we will list the differences between students and teachers regarding the problems solved in class. This part of our paper is based mainly on the learning theory we use (5) and on results of research on other courses $(2,7)$. The work we did on teacher problem-solving behavior (6) is in line with these results. The difficulties originate from two sources:

1) Teachers are experienced problem solvers (experts).

a) An expert takes steps in the problem-solving process in an abbreviated form. According to learning theory (5), a beginner must take most new steps in the problem-solving process explicitly and separately while an expert can take several steps at the same time.

b) The expert takes many steps only mentally. The phases in which this happens are analysis, planning problem-solving process, and evaluating the results. Usually, the only part written is the execution of routine operations (the calculations). The student, on the other hand, should take most steps on paper, especially at the beginning of the learning process (5)

c) The subject matter is easily accessible to the teacher (as an expert). Therefore, teachers may tend to underestimate the difficulties students have in finding their way around the subject matter. Even when this content is understood, its access may be difficult.

2) Almost all problems are routine for the teachers.

a) Because most problems are already standard problems for teachers, they skip in their own solution the transformation phase (steps $2 b$ and $c$ in Fig. 2). They must avoid skipping this when instructing their students. All too often teachers fall into the trap of underestimating the difficulty of solving quantitative problems. They tend to say "problem solving is math; they should learn this in their math lessons." The calculation phase (Fig. 2) is math, but the transformation process involves much more.

b) Although the strategy "working from the data" (forward reasoning) is rather efficient for solving standard problems, teachers, when explaining and working a problem on the blackboard, should avoid exclusive use of it. For solving problems which are real to the students, other strategies may offer a better chance of success. Students should be better oriented in their choice of strategy (6).

When teaching problem solving, teachers get little help from textbooks. The book we use, for example, does not show a systematic approach to solving a quantitative problem. This is unfortunately true for many of the texts, at least in our country. They pay little heed, if any, to the importance of a careful analysis of the problem, to the planning of the problem-solving steps, nor to the evaluation of the answer. Textbooks are written by experts too and therefore tend to miss the same points that many teachers do. 
In this respect, we are rather optimistic about the possibility of changing the behavior of teachers and of textbook writers. Once teachers are conscious of the fact that they, as experts, often omit steps in the problem-solving process that are essential to students, they tend to pay more attention to these steps, both in explanation and in exercises and feedback.

\section{Acknowledgment}

We gratefully acknowledge the useful suggestions and critical comments of Dr. H. Land, Dr. A. Pilot (both of Twente University of Technology) and of Dr. O. de Jong (University of Utrecht) on our work. Dr. H. Land and Mrs. Bea BanasSnijder (Laboratory School, Hengelo) gave useful comments on our use of the English language.

\section{Literature Cited}

(1) Mettes, C. T. C. W., Pilot, A., Roosink, H. and Kramers-Pals, H., (a) "Teaching and Learning Problem Solving in Science," Part I: “A General Strategy,” J. CHEM EDUC.,
57, 882 (1980); (b) Part II: "Learning Problem Solving in a Thermodynamics Course, J. CHEM. EDUC., 58, 51 (1981)

(2) Mettes C. T. S. W. and Pilot, A "Over het leren oplossen van natuurwetenschappelijke problemen (On Teaching and Learning Problem Solving in Science)" (Ph.D. thesis), Twente University of Technology, Enschede, The Netherlands, 1980, (in Dutch).

(3) Mettes, C. T. C. W. and Pilot, A., "Linking Factual and Procedural Knowledge in Solving Science Problems: A Case Study in a Thermodynamics Course," Instructional Science, 10, 333 (1981).

(4) Weeren, J. H. P. van, Kramers-Pals, H., de Mul, F. F. M., Peters, M. J. and Roosink, H. J., "Teaching Problem Solving in Physics-A Course in Electromagnetism," Enschede (Twente University of Technology), 1980 (to be published in Amer. J. Phys.).

(5) Talyzina N. F., "Psychological Bases of Programmed Instruction," Instructional Science, 2, 243 (1973).
Scina,

(6) Kramers-Pals, H., Lambrechts, J. and Wolff, P. J., "Conversion of Quantitative Problems in General Chemistry to Standard Problems," Enschede (Twente University of Technology)/Hengelo (Technical College), 1981.

(7) De Jong, O., "De probleemaanpak bij HAVO-leerlingen en hun leraren (The Problem Solving Behavior of High School Students and Their Teachers)," Utrecht (University of Utrecht), 1980 (in Dutch).

(8) Gilbert, G. L. "How do I get the answer?: Problem solving in chemistry," J. CHEM. EDUC., 57, 79 (1980).

(9) Larkin, J., McDermott, J., Simon, D. P., and Simon, H. A., "Expert and Novice Performance in Solving Physics Problems," Science, 208, 1335 (1980). 